\title{
PARADIGMI MISTI IN ALCUNI DIALETTI ITALO-ROMANZI: UN APPROCCIO MORFOLOGICO
}

\author{
PAVEL ŠTICHAUER \\ Università Carlo IV, Praga
}

\section{MIXED PARADIGMS IN SOME ITALO-ROMANCE VARIETIES: A MORPHOLOGICAL APPROACH}

This paper addresses the problem of mixed paradigms, i.e. cases of intraparadigmatic auxiliary alternation (esse/habere), as attested in some Italo-Romance varieties. It aims to show that, in some specific cases, auxiliary selection ceases to be motivated on syntactic grounds, where the traditional active/inactive verb classes are opposed, and begins to operate on a purely morphological basis. The paper represents one of the first attempts to deal with the issue of mixed paradigms in morphological terms. The aim is to show that auxiliary alternation within one and the same paradigm may follow both motivated and coherent patterns of distribution (e.g., singular versus plural) and unmotivated patterns of distribution, traditionally termed 'morphomic'. This article thus intends to put forward yet another case of morphologization of previously motivated distributional patterns.

Keywords: mixed paradigms; auxiliary selection; Italo-Romance varieties; inflection; periphrasis; lexical splits; morphomes; morphologization

Parole chiave: paradigmi misti; selezione dell'ausiliare; varietà italo-romanze; flessione; perifrasi; suddivisione lessicale; morfoma; morfologizzazione

\section{Introduzione}

Questo articolo ${ }^{1}$ rappresenta un primo tentativo, necessariamente ridotto alle sue linee principali, di considerare il fenomeno dei paradigmi misti in una prospettiva esclusivamente morfologica, sulla scia di quanto affermato, a varie riprese, da Loporca-

1 Il presente saggio rientra nell'ambito del progetto di ricerca GAČR 16-00236S (Paradigmi misti in alcune varietà italo-romanze), finanziato dalla Czech Science Foundation. Sono grato a Martin Maiden, Greville Corbet, Olivier Bonami e Adam Ledgeway per la discussione di alcune delle idee espresse in questa sede. Ringrazio anche Fabio Ripamonti per una revisione linguistica del presente testo. 
ro (2001: 461-462²; 2007: 186). Tali paradigmi misti, in cui l'alternanza dei due ausiliari HABERE / ESSE $(\mathrm{H} / \mathrm{E})^{3}$ si ritrova all'interno di uno stesso verbo, e non attraverso classi verbali come nell'italiano standard, sono ben attestati in diverse varietà italo-romanze centromeridionali, come nei dialetti marchigiani, abruzzesi, laziali e campani. Il paradigma che ricorre maggiormente, ad esempio, è quello in cui la prima e la seconda persona si oppongono alla terza nella selezione dell'ausiliare, come si vede nell'esempio (1), dove sono riportati i dati della varietà marchigiana di San Benedetto del Tronto (cf. Manzini - Savoia 2005, II: 682): ${ }^{4}$

(1)

\begin{tabular}{|c|c|c|}
\hline & SINGOLARE & PLURALE \\
\hline 1 & sว və่nu:tə & โعmə və'nu:tə \\
\hline 2 & Ji və'nu:tə & fetə və'nu:tə \\
\hline 3 & a və'nu:tə & a və'nu:tə \\
\hline
\end{tabular}

L'alternanza intraparadigmatica dei due ausiliari, in questo caso, $E$ nella prima e nella seconda del singolare e del plurale, $H$ nelle terze persone, all'interno del lessema in questione induce una suddivisione o partizione ('lexical split', cf. Corbett 2013; 2015; 2016) paragonabile a quelle indotte da una serie di alternanze tematiche tipiche dei verbi irregolari, in cui, come è ben noto, un dato tema verbale può ricorrere in un insieme di celle paradigmatiche (classe di partizione) che può anche non essere morfosintatticamente motivato o coerente (cf. Pirrelli - Battista 2000; Pirrelli 2000).

Lo scopo del presente articolo, auspicando che sia foriero di ulteriori sviluppi più approfonditi, è quello di esplorare l'ipotesi secondo la quale anche la selezione dell' ausiliare, una volta svincolata dall'originaria motivazione sintattica, possa morfologizzarsi e presentare, in seguito, vari schemi distribuzionali non motivati - morfomici (cf. Aronoff 1994), per i quali bisogna presupporre un livello di morfologia autonoma all'interno del sistema linguistico. Tale ipotesi si inserisce nel filone di ricerca sui fenomeni morfologici autonomi e, in particolare, prende spunto dalle osservazioni e dalle formulazioni sulle perifrasi espresse da Vincent (2011), Cruschina (2013) e Ledgeway (di prossima pubblicazione).

L'articolo è strutturato come segue. In (2) riassumo brevemente le ben note caratteristiche della selezione dell'ausiliare nelle lingue romanze standard e nei dialetti; in (3) procedo alla presentazione della nozione di paradigma misto; in (4) riporterò un campione di esempi di paradigmi sia motivati sia morfomici. In (5) mi soffermerò sulla presunta origine diacronica di tali sistemi misti; in (6) proporrò di trattare alcuni casi come chiari esempi di morfologizzazione, estendendo in tal modo la nozione tradizionale di questo processo. In (7) concluderò esplicitando le prospettive che in questo modo si aprono a una ricerca così impostata.

\footnotetext{
Si veda più avanti la nota 12 .

D'ora in poi, userò la forma etimologica latina HABERE / ESSE con le abbreviazioni in $\mathrm{H} / \mathrm{E}$ per i vari esiti dei due ausiliari, attestati in diverse varietà italo-romanze.

4 Evidenzierò in grigio le celle del paradigma accomunate dalla selezione dello stesso ausiliare.
} 


\section{La selezione dell'ausiliare nelle lingue romanze standard e nei dialetti}

Come è ben noto, le principali lingue romanze presentano una situazione variabile riguardo alla selezione dell' ausiliare. Vi è una distinzione assai netta tra le lingue ad un solo ausiliare generalizzato, come lo spagnolo o il catalano con $H$ (o anche il portoghese, che ha invece generalizzato TENERE 'ter'), e le lingue in cui la selezione dell'ausiliare avviene in base a vari criteri, soprattutto quello della cosiddetta intransitività scissa ('split intransitivity', cf. Bentley 2006), in cui, all'interno degli intransitivi, gli inaccusativi selezionano $E$, e gli inergativi propendono invece per $H$.

A livello delle varietà italo-romanze, la suddetta distinzione è ugualmente ben rappresentata. Esistono infatti varietà in cui si riscontra la generalizzazione dell'ausiliare $H, 5$ come nella varietà campana di Santa Maria a Vico (cf. Manzini - Savoia 2005, II: 779), cf. (2), oppure si può assistere alla generalizzazione dell' ausiliare $E,{ }^{6}$ come nella varietà marchigiana di Offida (cf. Manzini - Savoia 2005, II: 760), cf. (3):

(2)

\begin{tabular}{|l|l|l|}
\hline & SINGOLARE & PLURALE \\
\hline 1 & ad'dzu və' nutə & am' mu və' nutə \\
\hline 2 & a və'nutə & a'litə və' nutə \\
\hline 3 & a vvə'nutə & an' nu və' nutə \\
\hline
\end{tabular}

(3)

\begin{tabular}{|l|l|l|}
\hline & SINGOLARE & PLURALE \\
\hline 1 & so dər'mitə & semə dər'mitə \\
\hline 2 & je dər'mitə & setə dər'mitə \\
\hline 3 & $\varepsilon$ dər'mitə & $\varepsilon$ dər'mitə \\
\hline
\end{tabular}

La selezione dell'ausiliare dettata dalla classe verbale, in accordo con il criterio di intransitività scissa, si ritrova naturalmente anche in molte varietà settentrionali e centromeridionali, in cui si possono verificare anche ulteriori suddivisioni all'interno della classe dei riflessivi. Ad esempio, in molte varietà, i riflessivi indiretti selezionano $H$, anziché E come nell'italiano standard (cf. Manzini - Savoia 2005, II: 543; Loporcaro 2001: 462-468).

Tuttavia, tale situazione trasparente, in cui da una parte ritroviamo un solo ausiliare generalizzato e, dall'altra, l'alternanza viene regolata in base al principio sintattico di intransitività scissa, è lungi dall'essere l'unico modello distribuzionale dell'ausiliare. Di fatto, esistono diverse varietà in cui la sua selezione coinvolge altre categorie o tratti

5 In particolare si tratta di diverse varietà meridionali (cf. Manzini - Savoia 2005, II: 779-809).

6 La generalizzazione dell'ausiliare $E$ è invece tipica dei dialetti del Piemonte settentrionale e delle varietà di area mediana (cf. Manzini - Savoia 2005, II: 759-778). 
morfosintattici, come il modo (ad esempio, tra modi finiti ed infiniti), il tempo verbale, o addirittura la persona ed il numero (cf. Loporcaro 2001; 2007; 2014: 53; Ledgeway 2012: 321; Ledgeway 2014; Ledgeway: in stampa). Proprio nell'ambito dei sistemi di ausiliazione basati sulle distinzioni nella persona/numero si riscontrano modelli distribuzionali degni di un'ulteriore analisi.

\section{Paradigmi misti - le alternanze dell'ausiliare secondo la persona}

L'esempio (1) addotto all'inizio del presente articolo rappresenta un sistema che d'ora in poi chiameremo paradigma misto $^{7}$ dal momento che esibisce l'alternanza dei due ausiliari $H / E$ all'interno di uno stesso paradigma in base alla persona e al numero. Difatti, come abbiamo visto, la distribuzione dei due ausiliari non solo oppone la prima e la seconda persona alla terza, ma evidenzia anche l'opposizione tra il singolare e il plurale.

Recentemente tali sistemi person-based (o person-driven) sono stati oggetto di intense indagini, in particolare di impostazione generativista (cf., ad es., Manzini - Savoia 2005, II/III: cap. 5; D’Alessandro - Roberts 2010; Cennamo 2010; Legendre 2010; Loporcaro 2001; 2007; 2014; Ledgeway 2012: 317-327; in stampa). L'interesse per tali sistemi sta senza dubbio nel fatto le diverse distribuzioni, i patterns paradigmatici, si prestano ad una spiegazione morfosintatticamente coerente solo in alcuni casi, mentre in altri richiedono un approccio morfologico.

Prescindendo ora dalla situazione dei paradigmi del piuccheperfetto e del controfattuale, in cui la selezione dell'ausiliare tende a esibire la generalizzazione di uno dei due ausiliari, ${ }^{8}$ presenterò qui di seguito alcuni paradigmi che possono collocarsi in uno dei tre patterns morfologici ${ }^{9}$ che verranno definiti man mano che procederemo nella discussione dei dati: paradigma bilanciato, paradigma pāniniano, e paradigma strettamente morfomico.

\section{Paradigmi misti motivati e morfomici}

Il paradigma misto che abbiamo brevemente introdotto qui sopra viene indotto dalla selezione intraparadigmatica dei due ausiliari. In tal modo si crea, all'interno del lessema, una suddivisione o partizione lessicale ('lexical split') ${ }^{10}$ che apporta un elemento

\footnotetext{
$7 \mathrm{Mi}$ attengo, naturalmente, all'etichetta di mixed paradigm con cui il fenomeno viene descritto nella letteratura, cf., ad es., Bentley - Eythórsson 2001: 63-64.

8 Cf. Manzini - Savoia 2005, II: 681: "Un elemento di complessità ulteriore è introdotto dai paradigmi del piuccheperfetto, formati con l'imperfetto dell'ausiliare, nonché con i paradigmi del controfattuale, formato col condizionale e congiuntivo dell'ausiliare. Questi ultimi infatti presentano di norma la generalizzazione di uno dei due ausiliari."

9 Seguo qui la tipologia proposta da Bonami 2015: 69.

10 Cf. Corbett 2015: 146, n. 2: "At its simplest, a split is simply a division of a paradigm into parts (segments)."
} 
di complessità paragonabile, per esempio, all'effetto di un paradigma suppletivo come nel caso del verbo andare (combinato, diacronicamente, da due o più lessemi distinti).

In quel che segue, ricorrerò al termine tradizionale paradigma proprio nei casi in cui si riscontra la suddivisione appena definita. Inoltre, desidero rimandare ad un'ulteriore discussione, e in un'altra sede, l'analisi del paradigma misto più frequente che abbiamo esemplificato in (1). Tali sistemi sono stati esaminati sia dal punto di vista della diversa forza dei tratti morfosintattici coinvolti (ad esempio, l'opposizione tra le prime/seconde persone e le terze in termini di varie gerarchie della categoria di persona), sia dal punto di vista pragmatico, e non sintattico, in termini di opposizione tra partecipanti (la prima e la seconda persona) e non-partecipanti (la terza persona) all'atto linguistico (speech act participants / non-participants).

\subsection{Paradigmi bilanciati}

In alcuni casi, la distribuzione intraparadigmatica dei due ausiliari segue una suddivisione per così dire naturale, e quindi motivata, allorché oppone, all'interno di un paradigma, categorie morfosintatticamente ben delimitate. Tale opposizione è bilanciata quando il lessema viene suddiviso in due parti equiparabili11, come si vede nell' esempio (4), che riporta il paradigma di venire nella varietà abruzzese di Popoli (cf. Manzini - Savoia 2005, II: 688-689):

(4)

\begin{tabular}{|l|l|l|}
\hline & SINGOLARE & PLURALE \\
\hline 1 & səvə'niutə & a'vemmə və'niutə \\
\hline 2 & fi və'niutə & a've:tə və'niutə \\
\hline 3 & $\boldsymbol{\varepsilon}$ vvə'niutə & annə və'niutə \\
\hline
\end{tabular}

Uno 'split' bilanciato interessa una serie di fenomeni flessionali, come ad esempio le alternanze tematiche in latino, dove vengono opposti due temi diversi, quello del presente e quello del perfetto (ad es., $f a c-/ f \bar{e} c-$ ), oppure l'alternanza di due strategie di esponenza flessionale, l'una sintetica, l'altra perifrastica (come la differenza tra il presente sinteticamente espresso e il passato prossimo, formato per mezzo di una perifrasi).

\subsection{Paradigmi pāṇiniani}

Il termine 'pāṇiniano' si riferisce qui ad un fenomeno assai comune, che tavolta viene indicato anche con altre etichette (cf. Bonami 2015: 69). Per introdurre la nozione, mi attengo alla definizione proposta (e formalizzata) da Stump (2001: 22-23). Si tratta di una situazione in cui quando due regole entrano in concorrenza tra di loro viene data la precedenza alla regola più specifica, ristretta o limitata. Ad esempio, se la regola

11 Interpreto in questo modo la definizione del balanced split datane da Bonami 2015: 69, che richiede la suddivisione in base ad un tratto morfosintattico a due valori. In tal modo, la suddivisione del paradigma in base al numero è bilanciata solo quando il tratto del numero ha due valori (singolare / plurale). 
generale per la selezione dell'ausiliare impone la scelta di $H$ contemporaneamente alla selezione di $E$ in un insieme ristretto di celle paradigmatiche (o in un sottoinsieme di classi verbali), viene data la precedenza all'applicazione della regola più specifica per le celle coinvolte.

Nella fattispecie, molti paradigmi misti presentano una situazione in cui la regola generale stabilisce, per l'appunto, la scelta di $H$, tranne che in una sola cella del paradigma in questione, per la quale l'ausiliare corretto è invece $E$. In diverse varietà abruzzesi, infatti, si riscontrano distribuzioni in cui, ad esempio, la 2a pers. sg. presenta $E$ e il resto del paradigma esibisce invece $H$. Ma ci sono anche paradigmi in cui l'ausiliare $E$ coinvolge la 1a pers. sg., o invece soltanto la 3a pers. sg. (cf. Loporcaro 2001: 457; 2007: 184-185).

Il paradigma pāniniano può essere tipico anche di una sottoclasse verbale, come nel caso dei riflessivi in diverse varietà meridionali, si veda l'esempio (5) che riporta la parlata campana di Buonabitacolo (cf. Manzini - Savoia 2005, II: 653):

\begin{tabular}{|l|l|l|}
\hline & SINGOLARE & PLURALE \\
\hline 1 & m addza la'vato & nt amma la'vato \\
\hline 2 & t a la'vato & vaita la'vato \\
\hline 3 & s $\varepsilon$ la'vato/a & s anna la'vato \\
\hline
\end{tabular}

La sistemazione pāṇiniana rappresenta indubbiamente una situazione un po' meno prevedibile rispetto ai paradigmi bilanciati, in cui il lessema viene suddiviso in base a criteri morfosintattici coerenti. In questo caso, tuttavia, stabilire quale delle celle debba essere sotto il dominio della regola più specifica diventa alquanto problematico. In effetti, Loporcaro (2001: 457-459; 2007: 185), passando in rassegna alcuni dei tentativi di spiegare il fenomeno, conclude che le combinazioni possibili (anche all'interno della possibilità teorica di avere un paradigma pāniniano) sono di gran lunga più numerose, e sostiene anche che la loro concreta realizzazione attiene alla morfologia. ${ }^{12}$ La morfologia impone però un'organizzazione che può andare anche oltre ogni motivazione morfosintattica, fonologica o semantica. Le relazioni paradigmatiche, in cui necessariamente rientrano anche le perifrasi verbali, possono esibire distribuzioni assolutamente incoerenti, ovvero morfomiche.

\subsection{Paradigmi morfomici}

Il termine 'morfoma', dovuto ad Aronoff 1994, si è diffuso da tempo nell'ambito degli studi sui fenomeni autonomamente morfologici per il fatto che grazie ad esso è possibile studiare tutte quelle distribuzioni all'interno di un paradigma che non si

12 Cf. Loporcaro 2001: 461-462: "Se la selezione dell'ausiliare è un fatto eminentemente sintattico, il che appare difficile da negare, è plausibile che la ricerca sul tema, dovendo stabilire priorità, debba iniziare col mettere a fuoco, appunto, la funzionalità sintattica dell'ausiliazione, considerando solo in seconda battuta la concreta realizzazione morfo-lessicale di quest'ultima (sotto forma di questo o quello specifico verbo ausiliare). Ė questo un problema aggiuntivo, cui dedicare la massima attenzione, naturalmente, ma che attiene alla morfologia, non alla sintassi." 
prestano ad una spiegazione motivata (che sia fonologica, semantica o morfosintattica). Le distribuzioni morfomiche sono dunque interne alla morfologia non avendo, da una parte, alcun impatto sulla sintassi, e, dall'altra, alcuna motivazione semantica o fonologica (cf., ad es., Corbett 2015: 161-65; Bonami 2015: 69-70).

Tradizionalmente, le distribuzioni morfomiche sono state descritte ed analizzate per una nutrita serie di alternanze tematiche tipiche dei paradigmi verbali romanzi. Si deve a Maiden $(2005 ; 2011)$ la principale proposta terminologica di descrivere tali alternanze, sincronicamente prive di motivazione fonologica o semantica, per mezzo delle lettere L, $\mathrm{N}$ ed U per rappresentare spazialmente le classi di partizione coperte dalle celle paradigmatiche, occupate, appunto, da un dato allomorfo tematico. Le suddivisioni lessicali (nel senso di 'lexical split', come introdotto sopra) indotte da tali alternanze tematiche non sembrano essere le uniche possibilità teoriche: una suddivisione lessicale così imprevedibile ed irregolare può essere indotta anche dalla diversa selezione dell'ausiliare. È questa la nostra proposta principale che ora cercheremo di sviluppare.

Innanzitutto, è chiaro che la distribuzione intraparadigmatica dei due ausiliari, limitata qui, in modo deliberato (si veda sopra), soltanto ai paradigmi del passato prossimo, non corrisponde esattamente all'idea di Maiden. Infatti, le celle coinvolte in un pattern morfomico non si limitano ad un solo paradigma ma si estendono oltre, venendo ad occupare uno spazio che può essere descritto solo in termini di classe di partizione (cf. Pirrelli 2000: 53-54; Pirrelli - Battista 2000: 316-318). Pertanto, voler stabilire un pattern morfomico all'interno di uno stesso paradigma rappresenta necessariamente un tentativo ridotto ed incompleto che richiederà ulteriore analisi.

Prima di addurre qualche esempio di selezione dell'ausiliare che possa avvicinarsi notevolmente ad una distribuzione morfomica, dobbiamo introdurre un fattore importante, che complica il fenomeno dei paradigmi misti e di cui siamo costretti ad occuparci in un'altra sede. Si tratta della cosiddetta variazione libera dei due ausiliari, e cioè la situazione in cui, per un dato insieme di celle (o anche per un intero paradigma), diverse varietà consentono l'inserimento dell'uno o dell'altro ausiliare senza alcun impatto morfologico o semantico. La variazione libera dei due ausiliari, $\mathrm{H} \approx \mathrm{E}$, è stata oggetto di diverse analisi, tra cui spicca l'idea secondo la quale si abbia a che fare con due/multiple grammatiche in competizione (cf. Manzini - Savoia 2005, II: 740), oppure la proposta di trattare il fenomeno come un caso di ausiliazione tripla, in cui l'alternanza libera rappresenta una terza possibilità empirica di ausiliazione (Loporcaro 2007). Qualunque sia il quadro teorico che si adotti, resta il fatto che la variazione libera può interessare soltanto un sottoinsieme di celle paradigmatiche che possono anche non costituire una classe naturale, venendo così a creare, appunto, un modello morfomico paragonabile ai patterns definiti da Maiden $(2005 ; 2011)$.

Ci limiteremo in questa sede a proporre e a discutere un solo caso, che tra l'altro rappresenta eccezionalmente ${ }^{13}$ un paradigma del controfattuale (congiuntivo trapassato): quello in cui l'alternanza libera coinvolge tutte le persone del singolare e la terza persona del plurale, come si vede nell'esempio (6), dove viene riportato il verbo venire nella

13 Intendo qui eccezionale soltanto rispetto alla limitazione che mi sono imposto di trattare solo dei paradigmi del passato prossimo. In generale, infatti, i paradigmi misti sono tutt'altro che eccezionali negli altri tempi composti, anche se c'è una tendenza chiara alla generalizzazione di uno dei due ausiliari, come già menzionato sopra. 
varietà laziale di Viticuso (cf. Manzini - Savoia 2005, II: 706; si veda anche Ledgeway, in stampa: Sect. 3.2.1):

(6)

\begin{tabular}{|l|l|l|}
\hline & SINGOLARE & PLURALE \\
\hline 1 & fosse $\approx$ a'vessə mə'nutə & 'fussimə mə'nutə \\
\hline 2 & fusse $\approx$ a'vissə mə'nutə & 'fussitə mə'nutə \\
\hline 3 & fosse $\approx$ a'vessə mə'nutə & 'fossənə $\approx$ a'vissənə mə' nutə \\
\hline
\end{tabular}

Come si vede, la variazione libera dei due ausiliari (segnalata mediante $\approx$ ) interessa soltanto un sottoinsieme di celle paradigmatiche difficilmente spiegabile in termini fonologici o semantici: le tre persone del singolare e la terza del plurale non sembrano avere in comune alcun tratto semantico o morfosintattico che giustifichi la diversa selezione dell'ausiliare. Tuttavia, tale configurazione, denominata da Maiden (2005: 153; 2011: 242) N-pattern, rappresenta un modello paradigmatico molto forte che regola una serie di irregolarità morfologiche, indipendentemente dalla concreta realizzazione delle allomorfie, dai verbi con dittongo mobile (siedo-siedi-siede-sediamo-sedete-siedono) fino ai verbi altamente suppletivi come andare (vado, vai, va, andiamo, andate, vanno).

\section{L'origine diacronica dei sistemi misti}

Partendo dal presupposto che il sistema base di ausiliazione perfettiva sia quello tipico dell'italiano standard (cf. Bentley - Eythórsson 2001: 68), in cui la selezione dell' ausiliare contraddistingue due classi maggiori di verbi, inaccusativi e transitivi/inergativi, sorge spontanea la domanda riguardo all'origine diacronica di tali sistemi misti. Si tratta naturalmente di un quesito importante, ma che cercheremo soltanto di riformulare mettendo in discussione il punto di vista più diffuso e lasciando una spiegazione alternativa alla ricerca futura.

Si deve soprattutto a Bentley - Eythórsson (2001: 65) la proposta di uno scenario diacronico largamente condiviso (ma cf. Ledgeway: in stampa per un approccio diverso). L'ausiliazione mista sarebbe innescata dalla necessità di rimediare alla potenziale omonimia dell'ausiliare $H$, il quale tende a presentare esiti foneticamente identici nella $2^{\text {a }}$ e nella $3^{\text {a }}$ pers. del singolare $h a b e(s) / h a b e(t)>a i / a$. L'introduzione dell'ausiliare alternativo servirebbe dunque ad evitare tale omonimia, ripristinando in questo modo il contrasto paradigmatico tra le due persone. A sostegno di tale ipotesi i due studiosi adducono due elementi. In primo luogo, laddove si riscontra un sistema misto, esso presenta di norma l'ausiliare $E$ nella $2^{\text {a }}$ o nella $3^{\text {a }}$ persona. Tutti i sistemi misti hanno, infatti, in comune - indipendentemente da ulteriori microvariazioni - l'ausiliare $E$ proprio nella $2^{\text {a }}$ pers. del singolare (Bentley - Eythórsson 2001: 67; cf. anche l'utile schema in Manzini - Savoia 2005, II: 728). In secondo luogo, si sostiene che $E$ tenda poi a diffondersi in altre celle del paradigma, passando per varie fasi intermedie, tra cui anche l'alternanza libera, fino a generalizzarsi come unico ausiliare per l'intero paradigma indipendentemente dalla classe verbale e dal tipo di tempo composto. 
Tuttavia, ci sono vari punti che sembrano essere in contrasto con tale ipotesi. Innanzitutto, esistono numerose varietà italo-romanze, in particolare quelle settentrionali, che pur presentando un paradigma misto, esibiscono diverse sistemazioni dei due ausiliari in cui la $2^{\text {a }}$ pers. del singolare gioca un ruolo marginale (cf. lo schema in Manzini Savoia 2005, III: 14). Inoltre, è discutibile anche il peso della potenziale omonimia delle due persone. In effetti, in un numero cospicuo di dialetti, l'omonimia tra gli esiti delle due forme di $\mathrm{H}$ o viene tollerata (e sembra dunque essere innocua), o viene risolta in un modo diverso.

Infatti, numerose varietà rimediano all'omonimia in una maniera ben rappresentata anche nelle lingue romanze standard, e cioè introducono il pronome soggetto clitico in modo obbligatorio, come si vede nell'esempio (7), dove viene riportato il paradigma parziale di dormire nella varietà piemontese di Ala di Stura (cf. Manzini - Savoia 2005, II: 619):

\begin{tabular}{|l|lll|}
\hline & SINGOLARE & & \\
\hline 1 & ds & $\boldsymbol{\varepsilon}$ & dyr'mi \\
\hline 2 & t & a & dyr'mi \\
\hline 3 & ul & a & dyr' $\mathbf{m i}$ \\
\hline
\end{tabular}

Esiste anche un secondo modo, tipico di un gruppo di dialetti italo-romanzi, che consiste nella morfologizzazione del rafforzamento fonosintattico (RF) ai fini del mantenimento del contrasto tra le due persone, come si può vedere nell'esempio (8), ${ }^{14}$ che raccoglie i dati della varietà pugliese di Copertino (cf. Manzini - Savoia 2005, II: 624):

(8)

\begin{tabular}{|l|ll|}
\hline & SINGOLARE & \\
\hline 1 & addju & tur'mutu \\
\hline 2 & a & tur'mutu \\
\hline 3 & a & ddur'mutu \\
\hline
\end{tabular}

Il fatto che il RF possa morfologizzarsi in diversi modi per svolgere varie funzioni morfologiche è effettivamente ben documentato. Oltre al RF provocato dalla $3^{\text {a }}$ pers. sg. di $H$, attestato largamente in numerose varietà meridionali (cf. Torcolacci 2015: 11, 34), ci sono casi in cui il RF serve a segnalare l'opposizione tra la voce attiva di contro a quella passiva (ad es., nella varietà abruzzese di Arielli, so 'vistə / so v'vistə 'ho visto' / 'sono visto', cf. Biberauer - D’Alessandro 2006: 87-88, Torcolacci 2015: 31), o l'opposizione tra il maschile ed il femminile (ad es., in napoletano 'e figlie / 'e ffiglie 'i figli' / 'le figlie', cf. Ledgeway 2009: 47-48).

14 Si noti che anche l'esempio (2) riportato sopra corrisponde a questo schema. 


\section{Paradigma misto come un caso di morfologizzazione}

Quale che sia l'origine dei paradigmi misti, la selezione intraparadigmatica dell'ausiliare è un fenomeno che l'approccio sintattico può descrivere soltanto in maniera piuttosto limitata. Anche laddove la distribuzione rimane motivata morfosintatticamente (ad esempio, singolare versus plurale), l'ausiliare diventa un mezzo morfologico, ${ }^{15}$ "svincolato" per così dire dall'originaria motivazione sintattica - quella di codificare la fondamentale distinzione tra verbi inaccusativi e transitivi/inergativi.

In quanto mezzo morfologico, l'ausiliare - o la perifrasi formata per mezzo di esso rientra a pieno titolo nel novero dei fenomeni morfologici, come lo sono non soltanto i veri e propri esponenti di questo tipo (come le diverse desinenze flessionali), ma anche le alternanze tematiche che possono distribuirsi, come è ormai ben noto, in base a svariati schemi paradigmatici. È questo il senso corrente del termine morfologizzazione (cf. Pirrelli 2000: 3), che ora desidero estendere fino ad includere casi come questo. Intendo infatti proporre che il costituirsi di paradigmi misti venga percepito come un caso di morfologizzazione o, in generale, di ri-funzionalizzazione (cf. Pirrelli 2000: 158-160) di un'alternanza originariamente motivata - ma non a livello fonologico, bensì a livello sintattico, esattamente come afferma anche Pirrelli (2000: 205): "Ci siamo limitati a considerare la morfologizzazione in quanto rifunzionalizzazione morfologica di un'alternanza fonologica i cui contesti si sono opacizzati. Ma il fenomeno si estende alla rianalisi di unità indipendenti del contesto sintattico (ausiliari o preposizioni) come veri e propri esponenti morfologici di parola 'piena' come nel caso dell'evoluzione del FUTURO SEMPLICE italiano a partire da espressioni perifrastiche tardolatine. I due tipi di morfologizzazione sono molto diversi tra loro da molti punti di vista, ma c'è almeno un aspetto significativo che li accomuna: la reinterpretazione paradigmatica di una relazione base-derivato."

Se la selezione dell'ausiliare rappresenta, nelle lingue come l'italiano o il francese, un esempio sintattico del rapporto base-derivato, il paradigma misto rappresenta invece un caso in cui tale rapporto viene per così dire spezzato e, appunto, rianalizzato paradigmaticamente: i due ausiliari si distribuiscono all'interno del paradigma proprio come si comportano le desinenze flessionali oppure i temi allomorfi...

\section{Conclusioni e prospettive}

In questo articolo, ho voluto proporre un tentativo - perché allo stato attuale altro non è che un tentativo - di esaminare il fenomeno dei paradigmi misti attestati in numerose varietà italo-romanze dal punto di vista strettamente morfologico. Tale punto di vista non è assolutamente incompatibile con un approccio sintattico. Al contrario, il presente articolo vuole essere complementare all'approccio sintattico nella misura

15 Tale visione è propria, come abbiamo già avuto modo di vedere, non soltanto a Bentley - Eythórsson 2001 ma anche a Loporcaro 2001: 461-462, si veda sopra la n. 12; 2007: 186: "Of course, once we have described in these terms the syntax of auxiliation (...), we still have to specify where precisely (in which verb persons) the morphemes 'have' and 'be' occur. This is, however, a matter of morphology, not syntax, just like, say, the description of different personal endings within a paradigm." 
in cui mette in rilievo, sulla scia delle proposte di Loporcaro già menzionate sopra, la natura morfologica dei paradigmi misti. In base agli esempi riportati, si sostiene che anche a livello delle perifrasi verbali ci possano essere distribuzioni sia motivate sia morfomiche. Inoltre, il presente articolo sottolinea anche che le strategie di esponenza non si possono distinguere soltanto a livello di espressioni sintetiche $v s$ perifrastiche ma anche al livello interno alla perifrasi stessa. Naturalmente, come sempre accade, rimangono numerosi quesiti che ci ripromettiamo di affrontare in altra sede.

\section{BIBLIOGRAFIA}

Aronoff, M. (1994): Morphology by Itself. Stems and Inflectional Classes. Cambridge (MA): The MIT Press.

Bentley, D. (2006): Split Intransitivity in Italian. Amsterdam: John Benjamins.

Bentley, D. - Eythórsson, T. (2001): Alternation according to person in Italo-Romance. In: L. J. Brinton (ed.), Historical Linguistics 1999. Selected papers from the 14th International Conference on Historical Linguistics, Vancouver, 9-13 August 1999. Amsterdam: John Benjamins, pp. 63-74.

Biberauer, T. - D'Alessandro, R. (2006): Syntactic Doubling and the Encoding of Voice in Eastern Abruzzese. In: D. Baumer - D. Montero - M. Scanlon (eds.), Proceedings of the 25th West Coast Conference on Formal Linguistics. Somerville (MA): Cascadilla Proceedings Project, pp. 87-95.

Cennamo, M. (2010): Perfective auxiliaries in the pluperfect in some southern Italian dialects. In: R. D’Alessandro - A. Ledgeway - I. Roberts (eds.), Syntactic Variation: The dialects of Italy. Cambridge: Cambridge University Press, pp. 210-224.

Corbett, G. G. (2013): Periphrasis and possible lexemes. In: M. Chumakina - G. G. Corbett (eds.), Periphrasis. The Role of Syntax and Morphology in Paradigms. Proceedings of the British Academy, 180. Oxford: Oxford University Press, pp. 169-189.

Corbett, G. G. (2015): Morphosyntactic Complexity: A Typology of Lexical Splits. Language, 91, 1, pp. 145-193.

Corbett, G. G. (2016): Morphomic splits. In: A. R. Luís - R. Bermúdez-Otero (eds.), The morphome debate. Diagnosing and analysing morphomic patterns. Oxford: Oxford University Press, pp. 64-88.

Cruschina, S. (2013): Beyond the stem and inflectional morphology: an irregular pattern at the level of periphrasis. In: S. Cruschina - M. Maiden - J. C. Smith (eds.), The Boundaries of Pure Morphology. Oxford: Oxford University Press, pp. 262-283.

D’Alessandro, R. - Roberts, I. (2010): Past participle agreement: split auxiliary selection and the null-subject parameter. Natural Language and Linguistic Theory, 28, pp. 41-72.

Ledgeway, A. (2009): Grammatica diacronica del napoletano. Tübingen: Niemeyer.

Ledgeway, A. (2012): From Latin to Romance. Oxford: Oxford University Press.

Ledgeway, A. (2014): Romance Auxiliary Selection in Light of Romanian Evidence. In: G. Pană Dindelegan - R. Zafiu - A. Dragomirescu - I. Nicula - A. Nicolae (eds.), Diachronic Variation in Romanian. Newcastle: Cambridge Scholars Publishing, pp. 3-35.

Ledgeway, A. (in stampa): From Latin to Romance: The Great Leap. In: P. Crisma - G. Longobardi (eds.), The Oxford Handbook of Diachronic and Historical Linguistics. Oxford: Oxford University Press.

Ledgeway, A. (di prossima pubblicazione): From Coordination to Subordination: The Grammaticalisation of Progressive and Andative Aspect in the dialects of Salento. In: A. Cardoso - A. M. Martins - S. Pereira - C. Pinto - F. Pratas (eds.), Coordination and Subordination. Form and Meaning. Newcastle: Cambridge Scholars Publishing.

Loporcaro, M. (2001): La selezione dell'ausiliare nei dialetti italiani: dati e teorie. In: R. Sornicola E. Stenta Krosbakken - C. Stromboli (eds.), Dati empirici e teorie linguistiche: Atti del XXXIII Congresso della Società di Linguistica Italiana, Napoli, 28-30 ottobre 1999. Roma: Bulzoni, pp. 455-476.

Loporcaro, M. (2007): On triple auxiliation in Romance. Linguistics, 45, 1, pp. 173-222. 
Loporcaro, M. (2014): Perfective auxiliation in Italo-Romance. In: P. Benincà - A. Ledgeway - N. Vincent (eds.), Diachrony and Dialects. Grammatical Change in the Dialects of Italy. Oxford: Oxford University Press, pp. 48-70.

Maiden, M. (2005): Morphological autonomy and diachrony. In: G. Booji - J. van Marle (eds.), Yearbook of Morphology 2004. Dordrecht: Springer, pp. 137-175.

Maiden, M. (2011): Morphophonological innovation. In: M. Maiden - J. C. Smith - A. Ledgeway (eds.), The Cambridge History of the Romance Languages. Volume I. Structures. Cambridge: Cambridge University Press, pp. 216-267.

Manzini, R. - Savoia, L. (2005): I dialetti italiani e romanci: Morfosintassi generativa (I-III). Alessandria: Edizioni dell'Orso.

Pirrelli, V. - Battista, M. (2000): The paradigmatic dimension of stem allomorphy in Italian verb inflection. Italian Journal of Linguistics / Rivista di Linguistica, 12, 2, pp. 307-380.

Pirrelli, V. (2000): Paradigmi in morfologia. Un approccio interdisciplinare alla flessione verbale italiana. Pisa: Ist. Editoriali e Poligrafici.

Torcolacci, G. (2015): Marking the Default. Auxiliary selection in Southern Italian dialects. Tesi di dottorato discussa all'Università di Leida, 2015. Utrecht: LOT.

Vincent, N. (2011): Non-finite Forms, Periphrases, and Autonomous Morphology in Latin and Romance. In: M. Maiden - J. C. Smith - M. Goldbach - M.-O. Hinzelin (eds.), Morphological Autonomy. Perspectives from Romance Inflectional Morphology. Oxford: Oxford University Press, pp. 417-435.

Pavel Štichauer

Istituto di Studi Romanzi, Università Carlo

nám. Jana Palacha 2, 11638 Praha 1

pavel.stichauer@ff.cuni.cz 